\title{
SCHAUDER ESTIMATES FOR SOLUTIONS OF HIGHER-ORDER PARABOLIC SYSTEMS*
}

\author{
SERENA BOCCIA ${ }^{\dagger}$
}

\begin{abstract}
We prove global Schauder estimates for the derivatives of solutions to non-divergence form higher-order parabolic systems. All coefficients are taken only measurable in the time variable and Hölder continuous in the space variables. Moreover we require that the principal coefficients satisfy the so-called Legendre-Hadamard ellipticity condition. Using such estimates and some classical results, we also give a proof of existence and uniqueness for the Cauchy problem.
\end{abstract}

Key words. Parabolic systems, Schauder estimates, Hölder norms.

AMS subject classifications. 35K41, 35B45.

1. Introduction. This paper deals with the parabolic system

$$
u_{t}(t, x)+(-1)^{m} L u(t, x)=f(t, x),
$$

where the operator $L$ is defined as

$$
L=L(t, x)=\sum_{|\gamma| \leq 2 m} A^{\gamma}(t, x) D^{\gamma},
$$

$(t, x)$ in $(-\infty, T] \times \mathbb{R}^{d}$ if $T \in(-\infty, \infty)$ and in $\mathbb{R}^{d+1}$ if $T=\infty, m$ positive integer, $u$ and $f$ complex vector-valued functions, $A^{\gamma}$ complex matrix-valued function. Moreover the leading coefficients satisfy the so-called Legendre-Hadamard ellipticity condition, which is more general than the strong ellipticity condition considered, for example, in $[3,15]$ and is still stronger than the uniform parabolicity condition in the sense of Petrovskii, which was used in $[6,17,21]$.

The main aim of this investigation is to obtain a Hölder space estimate for the derivatives of the solutions $u$ of the above written system in terms of $f$ and $u$.

The Schauder (and similar) estimates play a fundamental role in the existence and regularity theory for linear and non-linear elliptic and parabolic equations and systems. In the literature derivations of these estimates are based on representations of solutions via Green's functions, used in [1, 7, 8, 21]; on the Campanato approach, introduced in [4], and used, for example, in [20]. The essence of Campanato's technique consists in a characterization of Hölder continuity via mean square oscillations and in obtaining the Schauder estimates for a general operator perturbing off the homogeneous, constant coefficient case. In this way it is possible to obtain estimates for the solution without resorting the direct estimate of the Newtonian potential as in the classical approach. A very nice exposition of this approach and its applications to various issues in the elliptic second-order theory can be found in the book by Giaquinta [9].

More recently, Safonov [19] has proved the Schauder estimates using the Harnack inequality of Krylov and Safonov [14]. Many of these Hölder estimates (with assumptions of continuity in the space and time for the derivatives) remain true even when the coefficients of the equation are not continuous in time (see, for instance,

\footnotetext{
*Received May 8, 2012; accepted for publication April 2, 2013.

$\dagger$ Dipartimento di Matematica, Università di Salerno, via Ponte Don Melillo, 84084 Fisciano (SA), Italy (seboccia@unisa.it).
} 
$[2,10,16])$. In particular, using maximum principle arguments, Brandt [2] showed that the second space derivatives of solutions of second order parabolic equations are Hölder in space and then Knerr [10] proved their Hölder continuity in time.

Some similar results were recently used in [13] for parabolic second-order equations with growing first-order coefficients to obtain the solvability in usual Hölder spaces (without weights).

In this paper we prove that each space derivative of solutions of system (1.1) admits a Hölder space estimate in $x$ and even in $t$ without $A^{\gamma}$ and $f$ being assumed to satisfy a Hölder condition in $t$. Such result extends to higher-order systems the results obtained by Brandt and Knerr for second-order equations.

When the leading coefficients are independent of $x$ and the lower-order coefficients are zero, the method relies on mean oscillation estimates of solutions to the systems. This approach was introduced by Krylov to deal with second-order elliptic and parabolic equations in the whole space, and can be found in his book [12]. For instance, by the mean oscillation estimate of $D^{2 m} u$ we mean a pointwise estimate of the form

$$
\begin{gathered}
\int_{Q_{r}}\left|D^{2 m} u-\left(D^{2 m} u\right)_{Q_{r}}\right| d x d t \\
\leq N k^{-1}\left(f_{Q_{k r}}\left|D^{2 m} u\right|^{2} d x d t\right)^{\frac{1}{2}}+N k^{m+\frac{d}{2}}\left(f_{Q_{k r}}|f|^{2} d x d t\right)^{\frac{1}{2}}
\end{gathered}
$$

for all $r \in(0, \infty)$ and $k \in\left[k_{0}, \infty\right)$, where $B_{r}$ is a ball with center in the origin and radius $r, Q_{r}=\left(-r^{2 m}, 0\right) \times B_{r}, k_{0}$ is a fixed integer. The mean oscillation estimates we prove follow by a particular case of a mean oscillation estimate contained in [5], similar to that in estimate (1.3), and are obtained by suitably adapting the techniques in $[12]$ to higher-order systems.

When the leading coefficients depend on $t$ and $x$ and the lower-order coefficients are zero, we use the method of freezing the coefficients. Afterwards some interpolation inequalities are used to obtain Schauder type estimates in the presence of lower-order terms. These results allow us to prove the solvability of the related Cauchy problem.

For future work we intend to generalize the results in [13] for higher-order systems. In particular, we intend to prove the following result for elliptic systems.

THEOREM. Let $A^{\gamma}$ be independent of $t$ and satisfy the assumptions stated in Section 2. Let $M u=\left(M u^{1}, \ldots, M u^{n}\right)$ be given by

$$
M u^{k}=a^{i j} D_{i} D_{j} u^{k}+b^{i} D_{i} u^{k}
$$

where $\left(a^{i j}\right)$ is a constant nonnegative definite matrix and $b^{i}$ are constant. Then there exists a constant $N$ depending only on $\delta, \alpha, K, n$, and $m$ (see Section 2), but independent of $a^{i j}$ and $b^{i}$ such that

$$
|u|_{2 m+\alpha} \leq N\left(|(L+M) u|_{\alpha}+|u|_{0}\right)
$$

for any $u \in C^{2 m+\alpha}\left(\mathbb{R}^{d}\right)$.

To the best of the author's knowledge this result is unknown even for single equations of second order. Even the following version of a slightly more general result 
than the above theorem seems to be unknown: in one dimensional case $(d=m=1$, $u$ real valued) we have

$$
[u]_{2+\alpha} \leq\left[a u^{\prime \prime}+b u^{\prime}+c u\right]_{\alpha},
$$

whenever $a, b$, and $c$ are constant and $a \geq 1$ and $c \leq 0$.

This result would be rather surprising since it states that the constants in the classical Schauder estimates for systems with constant coefficients are independent of the magnitudes of first and zero-order coefficients.

The proof of the above theorem we have in mind only works on the basis of the solvability of parabolic systems with coefficients measurable in time variable.

The paper is organized as follows. We introduce some notation and state the main results in the next section. Section 3 deals with some necessary auxiliary results. In Sections 4 and 5 we consider the case in which the leading coefficients of the operator $L$ are independent of $x$ and all other coefficients are zero. In particular, Section 4 is devoted to some preliminary mean oscillation estimates. In Section 5 we are able to prove our first main result, Theorem 2.1, after proving Lemma 5.1. In Section 6, we consider the more general case when the leading coefficients depend on $t$ and on $x$ and we prove our second main result, Theorem 2.4, that, together with some interpolation inequalities and with the results from Section 3, gives us Hölder space estimates in $x$ and in $t$ for the space derivatives of the solutions to our system. In the last section we state and prove an existence and uniqueness result for the Cauchy problem in a domain $\Omega=[0, T] \times \mathbb{R}^{d}$. Actually, to solve such problem, we divide the domain $\Omega$ into a finite number of domains $\left[\varepsilon_{s-1}, \varepsilon_{s}\right] \times \mathbb{R}^{d}$ with each $\varepsilon_{s}$ sufficiently small and smaller than $T$. First we solve the Cauchy problem in the smaller domains and then we find the solution of the Cauchy problem in the whole domain $\Omega$. A similar tecnique was used in [21].

Acknowledgement. Boccia conducted the research while visiting the School of Mathematics, University of Minnesota. The author is very grateful to N. V. Krylov for his invaluable insights, suggestions and guidance during the work. The author would also like to thank the referees for their useful comments.

2. Main result. We first introduce some notation used throughout the paper. A point in $\mathbb{R}^{d}$ is denoted by $x=\left(x_{1}, \ldots, x_{d}\right)$. For any $T \in(-\infty, \infty)$, we define

$$
\mathbb{R}_{T}^{d+1}=(-\infty, T] \times \mathbb{R}^{d}
$$

and, if $T=\infty$, we set $\mathbb{R}_{T}^{d+1}=\mathbb{R}^{d+1}$. A point in $\mathbb{R}_{T}^{d+1}$ is denoted by $X=(t, x)$.

We set $B_{r}(x)=\left\{y \in \mathbb{R}^{d}:|x-y|<r\right\}, B_{r}=B_{r}(0), Q_{r}(t, x)=\left(t-r^{2 m}, t\right) \times B_{r}(x)$ and $Q_{r}=Q_{r}(0,0)$. Denote

$$
u_{Q_{r}(t, x)}=\int_{Q_{r}(t, x)} u(s, y) d y d s=\frac{1}{\left|Q_{r}(t, x)\right|} \int_{Q_{r}(t, x)} u(s, y) d y d s,
$$

the average value of a function $u(s, y)$ over $Q_{r}(t, x)$ and

$$
u_{B_{r}(x)}(t)=\int_{B_{r}(x)} u(t, y) d y=\frac{1}{\left|B_{r}(x)\right|} \int_{B_{r}(x)} u(t, y) d y
$$

the average value of a function $u(t, y)$ over $B_{r}(x)$. 
Now we specify some notations in (1.1) and (1.2). The symbol $\gamma$ denotes a multiindex, i.e.,

$$
\begin{gathered}
\gamma=\left(\gamma_{1}, \ldots, \gamma_{d}\right), \quad \gamma_{i} \geq 0 \quad \forall i=1, \ldots, d, \quad|\gamma|=\gamma_{1}+\ldots+\gamma_{d} \\
D^{\gamma}=D_{1}^{\gamma_{1}} \ldots D_{d}^{\gamma_{d}}, \quad D_{i}^{\gamma_{i}}=\frac{\partial^{\gamma_{i}} u}{\partial x_{i}^{\gamma_{i}}} \quad \forall i=1, \ldots, d, \quad u_{t}=\frac{\partial u}{\partial t}
\end{gathered}
$$

Sometimes, if $\gamma$ is a multi-index of length $k$, we use the notation $D^{k}$ to denote $D^{\gamma}$.

For each $\gamma, A^{\gamma}=\left[A_{i j}^{\gamma}(t, x)\right]_{i, j=1}^{n}$ is an $n \times n$ complex matrix-valued function. The involved functions are complex vector-valued functions, that is,

$$
u=\left(u^{1}, \ldots, u^{n}\right)^{t r}, f=\left(f^{1}, \ldots, f^{n}\right)^{t r} .
$$

Moreover, we put

$$
L_{0}=L_{0}(t, x)=\sum_{|\gamma|=2 m} A^{\gamma}(t, x) D^{\gamma} .
$$

In the first part of the paper we will be fixing our attention on the system

$$
u_{t}(t, x)+(-1)^{m} L_{0} u(t, x)=f(t, x), \quad(t, x) \in(-\infty, T) \times \mathbb{R}^{d} .
$$

For $k=0,1,2, \ldots$, as usual, we denote $C_{l o c}^{k}\left(\mathbb{R}^{d}\right)$ the set of all functions $u=u(x)$ whose derivatives $D^{\gamma} u$ for $|\gamma| \leq k$ are continuous in $\mathbb{R}^{d}$. We set

$$
|u|_{0}=\sup _{\mathbb{R}^{d}}|u|, \quad[u]_{k}=\max _{|\gamma|=k}\left|D^{\gamma} u\right|_{0}
$$

The space $C^{k}\left(\mathbb{R}^{d}\right)$ is the Banach space of all functions $u \in C_{l o c}^{k}\left(\mathbb{R}^{d}\right)$ for which the following norm

$$
|u|_{k}=\sum_{j=0}^{k}[u]_{j}
$$

is finite. If $\alpha \in(0,1)$, we call $u$ Hölder continuous with exponent $\alpha$ if the seminorm

$$
[u]_{\alpha}=\sup _{\substack{x, y \in \mathbb{R}^{d} \\ x \neq y}} \frac{|u(x)-u(y)|}{|x-y|^{\alpha}}
$$

is finite. We set

$$
[u]_{k+\alpha}=\max _{|\gamma|=k}\left[D^{\gamma} u\right]_{\alpha}
$$

and $C^{k+\alpha}\left(\mathbb{R}^{d}\right)$ is the space of functions $u \in C^{k}\left(\mathbb{R}^{d}\right)$ such that

$$
|u|_{k+\alpha}=|u|_{k}+[u]_{k+\alpha}
$$

is finite.

For any function $f(t, x)$, defined for $x$ in $\mathbb{R}^{d}$ and for $t$ in some range, we denote

$$
\begin{array}{r}
|f|_{0}(t)=|f(t, \cdot)|_{0}, \quad|f|_{k}(t)=|f(t, \cdot)|_{k} \\
{[f]_{\alpha}(t)=[f(t, \cdot)]_{\alpha}, \quad|f|_{k+\alpha}(t)=|f(t, \cdot)|_{k+\alpha},}
\end{array}
$$


which are functions of the time variable.

Let $T \in(-\infty, \infty]$ and $S \in[-\infty, T)$. We will be working with the set $\mathcal{C}^{2 m+\alpha}(S, T)$ of functions $u(t, x)$ defined for all finite $t \in[S, T]$ and $x \in \mathbb{R}^{d}$ such that

(i) the function $u$ is continuous in its domain;

(ii) for each finite $S \leq t \leq T$, we have $u(t, \cdot) \in C^{2 m+\alpha}\left(\mathbb{R}^{d}\right)$ and $|u|_{2 m+\alpha}(t)$ is bounded in $t$;

(iii) there is a measurable function $g(t, x)$ defined on the domain of the function $u$ such that for any $\zeta \in C_{0}^{\infty}(\mathbb{R})$ the function $\zeta(t) g(t, x)$ is bounded and $\alpha$ - Hölder continuous in $x$ with constant independent of $t$ and for any $x \in \mathbb{R}^{d}$ and any finite $s$ and $t$, such that $S \leq s<t \leq T$, we have

$$
u(t, x)-u(s, x)=\int_{s}^{t} g(r, x) d r .
$$

For such a function $u$ we denote $u_{t}=g$. Obviously $g$ is the generalized derivative of $u$ with respect to $t$. By $\mathcal{C}^{2 m+\alpha}(T)$ we denote the space $\mathcal{C}^{2 m+\alpha}(S, T)$ when $S=-\infty$.

Recall that a continuous function $u$ has a bounded generalized derivative with respect to a coordinate if and only if it is Lipschitz continuous with respect to this coordinate and if and only if $u$ is absolutely continuous with respect to the coordinate and its classical derivative (existing almost everywhere) is bounded. Under any of the above conditions the classical derivative coincides with the generalized one and its essential supremum equals the Lipschitz constant.

The solution of the Cauchy problem (which we will consider in the last section) will be looked for in $\mathcal{C}^{2 m+\alpha}(0, T)$ and in this class (1.1) is equivalent to the fact that, for any $x \in \mathbb{R}^{d}$ and finite $0 \leq s<t \leq T$, we have

$$
u(t, x)-u(s, x)=\int_{s}^{t} f(r, x) d r-(-1)^{m} \int_{s}^{t} L u(r, x) d r .
$$

In addition to the well known spaces $L_{p}$ and $W_{p}^{k}$, we introduce the following function spaces:

$$
W_{p}^{1,2 m}\left(\mathbb{R}^{d+1}\right)=\left\{u: u_{t}, D^{\gamma} u \in L_{p}\left(\mathbb{R}^{d+1}\right),|\gamma| \leq 2 m\right\}
$$

equipped with its natural norm.

For any $T \in(-\infty, \infty]$, we define the parabolic distance between two points $(t, x),(s, y) \in \mathbb{R}_{T}^{d+1}$ with finite $t, s$ in the following way:

$$
\langle(t-s, x-y)\rangle:=|t-s|^{\frac{1}{2 m}}+|x-y| .
$$

If $f$ is a function defined on $\mathbb{R}_{T}^{d+1}$ we denote

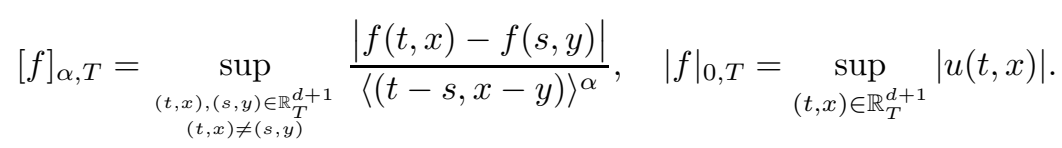

By $C^{\frac{\alpha}{2 m}, \alpha}\left(\mathbb{R}_{T}^{d+1}\right)$ we denote the space of all functions $f$ for which $[f]_{\alpha, T}+|f|_{0, T}<\infty$.

Let $\delta, K>0$ be two constants. We assume that all coefficients are measurable in $\mathbb{R}^{d+1}$, complex valued, bounded,

$$
\left|A^{\gamma}(t, x)\right| \leq \delta^{-1}, \quad \forall \gamma,|\gamma| \leq 2 m, \quad \forall(t, x) \in \mathbb{R}^{d+1},
$$


and, for some $\alpha \in(0,1)$,

$$
\left[A^{\gamma}\right]_{\alpha}(t) \leq K \quad \forall \gamma,|\gamma| \leq 2 m, \quad \forall t \in \mathbb{R}
$$

In addition, we impose the Legendre-Hadamard ellipticity condition on the leading coefficients (see, for instance, [8], [9]). Here we call $A^{\gamma}$ the leading coefficients if $|\gamma|=$ $2 m$. All the other coefficients are called lower-order coefficients. By the LegendreHadamard ellipticity condition we mean

$$
\Re\left(\sum_{|\gamma|=2 m} \theta^{t r} \xi^{\gamma} A^{\gamma}(t, x) \bar{\theta}\right) \geq \delta|\xi|^{2 m}|\theta|^{2}
$$

for all $(t, x) \in \mathbb{R}^{d+1}, \xi \in \mathbb{R}^{d}$, and $\theta \in \mathbb{C}^{n}$. Here we use $\Re(f)$ to denote the real part of $f$ and $\xi^{\gamma}$ to denote $\left(\xi_{1}^{\gamma_{1}}\right) \cdot \ldots \cdot\left(\xi_{d}^{\gamma_{d}}\right)$.

From now on, we always suppose that our previous hypotheses on the coefficients are satisfied. Let $T \in(-\infty, \infty]$ and $\alpha \in(0,1)$ be the number for which hypothesis (2.8) holds.

Note that the notation $\sup _{t \leq T} f(t)$ we often deal with in the following has the usual meaning if $T$ is finite and means the upper bound over all finite $t$ if $T=\infty$.

Here are our main results.

THEOREM 2.1. If the coefficients of the operator $L_{0}$ depend only on $t$ and $u \in$ $\mathcal{C}^{2 m+\alpha}(T)$ satisfies (2.2), then for all $(t, x),(s, y) \in \mathbb{R}_{T}^{d+1}$ with finite $t, s$ and for all $\gamma$ such that $|\gamma|=2 m$, we have

$$
\left|D^{\gamma} u(t, x)-D^{\gamma} u(s, y)\right| \leq N \sup _{\bar{t} \leq T}[f]_{\alpha}(\bar{t})\langle(t-s, x-y)\rangle^{\alpha},
$$

where $N=N(d, \alpha, n, m, \delta)$.

REMARK 2.2. This is a basic Hölder space estimate for parabolic systems. A somewhat unusual feature of it is that $D^{2 m} u$ admit a Hölder space estimate even in $t$ without requiring $f$ and the coefficients of the operator to satisfy a Hölder condition in $t$. Of course, if the leading coefficients and $f$ are $C^{\frac{\alpha}{2 m}}$ Hölder in $t$ uniformly respect to $x$, then from the equation $u_{t}=f-(-1)^{m} L_{0} u$ we see that $u_{t}$ is also $C^{\frac{\alpha}{2 m}}$ Hölder in $t$ and $C^{\alpha}$ Hölder in $x$, which gives a standard basic Hölder space estimates for parabolic systems.

REMARK 2.3. One can ask a natural question as to weather Theorem 2.1 can be extended to bounded domains. Unfortunately, it cannot. Even in one space dimension for the scalar heat equation $u_{t}=D^{2} u+I_{t<1}$ in $\{x \in(0,1)\}$ with boundary data $u(t, 0)=0$ the function $D^{2} u$ cannot be Hölder continuous in $x$ with constant independent of $t$ because by our Corollary 3.3 it would be then Hölder continuous with respect to $t$ but $I_{t \leq 1}=u_{t}(t, 0)-D^{2} u(t, 0)=D^{2} u(t, 0)$ and the left-hand side is not even continuous.

THEOREM 2.4. Let $u \in \mathcal{C}^{2 m+\alpha}(T)$ satisfy (1.1). Then there exists a constant $N=N(K, d, \alpha, n, m, \delta)$ such that

$$
\sup _{s \leq T}|u|_{2 m+\alpha}(s) \leq N \sup _{t \leq T}\left([f]_{\alpha}(t)+|f|_{0}(t)+|u|_{0}(t)\right) .
$$


REMARK 2.5. As consequence of Theorem 2.4, by means of interpolation inequalities, we will obtain an a priori estimate analogous to (2.10) for the solutions to (1.1). Such estimate will be necessary to use the classical method of continuity and, then, to solve our Cauchy problem.

3. An embedding theorem. This section is devoted to prove a result, that is, actually, an embedding theorem which has little to do with parabolic systems. However it is very useful in order to show that, under suitable hypotheses about the function $u$, the derivatives $D^{2 m} u$ in $x$ are Hölder continuous with respect to the time variable.

Before to state and prove the theorem, we need of the following elementary result.

In the sequel $k$ denotes a fixed positive integer number.

LEMMA 3.1. Let $V$ be a convex closed round cone in $\mathbb{R}^{d}$ with vertex at the origin and nonempty interior. Let $u^{\gamma}$ be a collection of real numbers varying the multi-index $\gamma$ of length $k$. Then, for any $\gamma,|\gamma|=k$, there is a constant $N$, independent of $u^{\gamma}$, such that

$$
\left|u^{\gamma}\right| \leq N \max _{|\xi|=1, \xi \in V}\left|\sum_{|\eta|=k} u^{\eta} \xi^{\eta}\right| .
$$

Proof. By contradiction, we assume that for each $n \in \mathbb{N}$, exists a sequence $\left\{u_{n}^{\gamma}\right\}$ such that

$$
\max _{|\gamma|=k}\left|u_{n}^{\gamma}\right| \geq n \max _{|\xi|=1, \xi \in V}\left|\sum_{|\eta|=k} u_{n}^{\eta} \xi^{\eta}\right| .
$$

Without loss of generality, we can assume that

$$
\max _{|\gamma|=k}\left|u_{n}^{\gamma}\right|=1
$$

Then from (3.2) and (3.3) we obtain

$$
\max _{|\xi|=1, \xi \in V}\left|\sum_{|\eta|=k} u_{n}^{\eta} \xi^{\eta}\right| \leq \frac{1}{n} .
$$

By the boundedness of the sequence $u_{n}^{\gamma}$, we deduce that there exists a subsequence, still denoted by $u_{n}^{\gamma}$, such that

$$
\lim _{n \rightarrow+\infty} u_{n}^{\gamma}=u^{\gamma} \quad \forall \gamma,|\gamma|=k .
$$

Passing to the limit in (3.3) and in (3.4) we get, respectively,

$$
\begin{gathered}
\max _{|\gamma|=k}\left|u^{\gamma}\right|=1, \\
\sum_{|\eta|=k} u^{\eta} \xi^{\eta}=0 \quad \forall \xi \in V,|\xi|=1 .
\end{gathered}
$$

We show that (3.7) implies that

$$
u^{\eta}=0 \quad \forall \eta,|\eta|=k
$$


and this is a contradiction. In order to prove (3.8), denote

$$
f(\xi)=\sum_{|\eta|=k} u^{\eta} \xi^{\eta}
$$

and observe that, using (3.7), we can say that for any multi-index $\eta$,

$$
D^{\eta} f(\xi)=0 \quad \forall \xi \in V,|\xi|=1 .
$$

In particular, if $\xi \in V$ such that $|\xi|=1$ and $\eta$ is a multi-index of length $k$, we have

$$
D^{\eta} f(\xi)=\eta ! u^{\eta}=0
$$

and so (3.8). The proof is complete.

Take $\gamma$ and $V$ as in Lemma 3.1, take an $h>0$, and consider the truncated cone

$$
V_{h}=V \cap\left\{x:|x| \leq \frac{k}{2} h\right\} .
$$

The spaces $C^{\alpha}\left(V_{h}\right)$ are defined in the same way as $C^{\alpha}\left(\mathbb{R}^{d}\right)$. We also write $[\cdot]_{\alpha, V_{h}}$ to denote the usual Hölder seminorm in $C^{\alpha}\left(V_{h}\right)$. In the same way we introduce the spaces $C^{k+\alpha}\left(V_{h}\right)$ and the seminorms $[\cdot]_{k+\alpha}$. Notice that $V_{h}$ is a closed set. In particular, the functions from $C^{k+\alpha}\left(V_{h}\right)$ are $k$-times continuously differentiable in the interior of $V_{h}$ and their derivatives admit continuous extension to the boundary of $V_{h}$. In the following lemma by $D^{\gamma} u(r, 0)$ we mean these continuations.

THEOREM 3.2. Let $u:\left[0, h^{k}\right] \times V_{h} \rightarrow \mathbb{R}$ be a continuous function such that $u(t, \cdot) \in C^{k+\alpha}\left(V_{h}\right), t \in\left[0, h^{k}\right]$, and assume that there exists a function $g(t, x)$ defined on $\left[0, h^{k}\right] \times V_{h}$ such that $g(t, \cdot) \in C^{\alpha}\left(V_{h}\right), t \in\left[0, h^{k}\right]$, and (2.5) is verified for $0 \leq s \leq$ $t \leq h^{k}, x \in V_{h}$ (we set $u_{t}=g$ ).

Then there is a constant $N>0$, independent of $h$ and $u$ such that, for any $\gamma$, $|\gamma|=k$,

$$
\left|D^{\gamma} u\left(h^{k}, 0\right)-D^{\gamma} u(0,0)\right| \leq N I_{h} h^{\alpha},
$$

where

$$
I_{h}:=\sup _{r \in\left[0, h^{k}\right]}\left(\left[u_{t}\right]_{\alpha, V_{h}}(r)+[u]_{k+\alpha, V_{h}}(r)\right) .
$$

Proof. The parabolic dilation $(s, x) \rightarrow\left(\left(2^{-1} h\right)^{k} s, 2^{-1} h x\right)$ allows us to assume that $h=2$.

Next assume that the first basis vector $l$ is inside $V_{2}=V_{h}$. After denoting with $T_{y}$ the operator $u(t, x) \rightarrow u(t, x+y)$ and with $\gamma_{1}=(k, 0, \ldots, 0)$, we can write

$$
\begin{aligned}
\left|D^{\gamma_{1}} u\left(2^{k}, 0\right)-D^{\gamma_{1}} u(0,0)\right| & \leq\left|D^{\gamma_{1}} u\left(2^{k}, 0\right)-\left[\left(T_{l}-1\right)^{k} u\left(2^{k}, 0\right)\right]\right| \\
& +\left|D^{\gamma_{1}} u(0,0)-\left[\left(T_{l}-1\right)^{k} u(0,0)\right]\right|+I
\end{aligned}
$$

where

$$
I=\left|\left[\left(T_{l}-1\right)^{k} u\right]\left(2^{k}, 0\right)-\left[\left(T_{l}-1\right)^{k} u\right](0,0)\right| .
$$


Before estimating the quantities in (3.13), we need to observe some facts.

By induction we prove that, for each $(t, x)$ in $\left[0, h^{k}\right] \times V_{2}$,

$$
\left(T_{l}-1\right)^{k} u(t, x)=\left(D^{\gamma_{1}} u\right)(t, x+\theta l), \quad 0 \leq \theta \leq k .
$$

When $k=1$, our aim is to prove that

$$
\left(T_{l}-1\right) u(t, x)=\left(D^{1} u\right)(t, x+\theta l), \quad 0 \leq \theta \leq 1,
$$

and this easily follows by Lagrange Theorem.

Now consider $k>1$ and suppose that (3.15) holds for $k-1$, we want to prove that holds for $k$. If we denote

$$
V(t, x)=\left(T_{l}-1\right)^{k-1} u(t, x)
$$

by (3.16) and the induction hypotesis, we have

$$
\begin{aligned}
& \left(T_{l}-1\right)^{k} u(t, x)=\left(T_{l}-1\right) V(t, x)=\left(D^{1} V\right)(t, x+\theta l) \\
& =\left(T_{l}-1\right)^{k-1}\left(D^{1} u\right)(t, x+\theta l)=D^{\gamma_{2}}\left(D^{1} u\right)\left(t, x+\theta l+\theta_{1} l\right)
\end{aligned}
$$

where $0 \leq \theta \leq 1,0 \leq \theta_{1} \leq k-1$ and $\gamma_{2}=(k-1,0, \ldots, 0)$. From the last equality, we easily get (3.15).

Then, using (3.15), we obtain

$$
\begin{aligned}
\left|D^{\gamma_{1}} u\left(2^{k}, 0\right)-\left[\left(T_{l}-1\right)^{k} u\left(2^{k}, 0\right)\right]\right| & =\left|D^{\gamma_{1}} u\left(2^{k}, 0\right)-D^{\gamma_{1}} u\left(2^{k}, \theta_{1} l\right)\right| \\
& \leq C_{1}\left[D^{\gamma_{1}} u\left(2^{k}, \cdot\right)\right]_{\alpha, V_{2}}
\end{aligned}
$$

and

$$
\begin{aligned}
\left|D^{\gamma_{1}} u(0,0)-\left[\left(T_{l}-1\right)^{k} u(0,0)\right]\right| & =\left|D^{\gamma_{1}} u(0,0)-D^{\gamma_{1}} u\left(2^{k}, \theta_{2} l\right)\right| \\
& \leq C_{2}\left[D^{\gamma_{1}} u\left(2^{k}, \cdot\right)\right]_{\alpha, V_{2}},
\end{aligned}
$$

where $C_{1}, C_{2}$ depend only on $k, 0 \leq \theta_{1}, \theta_{2} \leq k$.

Now we estimate $I$. First we note that for each function $v(t, x)$, defined on $\left[0, h^{k}\right] \times V_{2}$,

$$
\left|\left(T_{l}-1\right) v(t, x)\right| \leq 2 \max \{|v(t, y)|: x \leq y \leq x+l\}
$$

Using (3.21), by induction, we can easily see that, for any $k>1$,

$$
\left|\left(T_{l}-1\right)^{k} v(t, x)\right| \leq 2^{k} \max \{|v(t, y)|: x \leq y \leq x+k l\} .
$$

Observe that, by the linearity of the operator and Newton-Leibnitz formula, it follows that for any function $c$ independent of $x$

$$
I=\int_{0}^{2^{k}}\left[\left(T_{l}-1\right)^{k} u_{t}\right](r, 0) d r=\int_{0}^{2^{k}}\left[\left(T_{l}-1\right)^{k}\left(u_{t}-c\right)\right](r, 0) d r .
$$

Choosing $c=u_{t}(r, 0)$ and using (3.22), from the last equality, we get

$$
I \leq 2^{2 k} \sup _{\substack{0 \leq x \leq k l \\ 0 \leq r \leq 2^{k}}}\left|u_{t}(r, x)-u_{t}(r, 0)\right| \leq C_{3} \sup _{r \in\left[0,2^{k}\right]}\left[u_{t}(r, \cdot)\right]_{\alpha, V_{2}},
$$


where $C_{3}$ depends only on $k$.

Upon combining the above estimates, we come to

$$
\left|D^{\gamma_{1}} u\left(2^{k}, 0\right)-D^{\gamma_{1}} u(0,0)\right| \leq N I_{2} .
$$

Since by an orthonormal change of coordinates any unit vector $\xi \in V_{2}$ can be transformed into the first basis vector, we have the previous estimate proved also for the $k$-order directional derivative of $u$ along $\xi$, i.e.,

$$
\left|\sum_{|\gamma|=k}\left(\xi^{\gamma} D^{\gamma} u\left(2^{k}, 0\right)-\xi^{\gamma} D^{\gamma} u(0,0)\right)\right| \leq N I_{2}
$$

for all unit $\xi \in V_{2}$ and for any multi-index $\gamma,|\gamma|=k$.

The result now follows from Lemma 3.1.

Corollary 3.3. Let $S$ be in $[-\infty, T)$. For any $u \in \mathcal{C}^{k+\alpha}(S, T)$ and finite $S \leq$ $t, s \leq T, x \in \mathbb{R}^{d}$ and $\gamma,|\gamma|=k$ we have

$$
\left|D^{\gamma} u(t, x)-D^{\gamma} u(s, x)\right| \leq N I|t-s|^{\alpha / k},
$$

where $N$ is independent of $u, t, s, x$ and

$$
I=\sup _{r \in[s, t]}\left(\left[u_{t}(r, \cdot)\right]_{\alpha}+[u(r, \cdot)]_{k+\alpha}\right) .
$$

Proof. It is sufficient to apply Theorem 3.2 denoting $t-s=h^{k}$ and shifting the origin in $\mathbb{R}^{d+1}$ to point $(s, x)$.

REMARK 3.4. If we come back to our system, Corollary 3.3 allows us to conclude that if the solution $u$ of (1.1) belongs to $\mathcal{C}^{2 m+\alpha}(S, T)$ then the derivatives $D^{2 m} u$ are also Hölder continuous in $t$ with exponent $\alpha / 2 m$. Moreover, the Hölder seminorm in $t$ of each space derivative of order less or equal than $2 m$ is bounded by

$$
N \sup _{r}\left(\left[u_{t}(r, \cdot)\right]_{\alpha}+|u(r, \cdot)|_{2 m+\alpha}\right) .
$$

Here the constant $N$ being independent of $u$ depends only on $d, \alpha, n$, and $m$. However, the author does not know any way how to compute this constant.

In connection to this, is also worth noting that if $u \in \mathcal{C}^{2 m+\alpha}(S, T)$, then $u$ is locally Lipschitz in $(t, x)$ since, by the definition of such space, the derivative $D u$ is bounded and $u_{t}$ is locally bounded.

It is noteworthy that, for example, the estimate (3.27) may not be as useful as estimates as in (2.9) where information about the constant $\mathrm{N}$ is known.

4. Auxiliary results. Throughtout the next two sections we consider only the case when the leading coefficients are independent of $x$ and the lower-order coefficients are zero.

From Corollary 2 in [5] we obtain the following

TheOREM 4.1. Let $r \in(0, \infty), k \in[8, \infty), X_{0}=\left(t_{0}, x_{0}\right) \in \mathbb{R}^{d+1}$ and $f \in$ $L_{2, \text { loc }}\left(\mathbb{R}^{d+1}\right)$. Assume that $u \in W_{2, \text { loc }}^{1,2 m}\left(\mathbb{R}^{d+1}\right)$ satisfies

$$
u_{t}+(-1)^{m} L_{0} u=f \quad \text { in } Q_{k r}\left(X_{0}\right) .
$$


Then for any $\gamma,|\gamma|=2 m$, we have

$$
\begin{aligned}
f_{Q_{r}\left(X_{0}\right)}\left|D^{\gamma} u-\left(D^{\gamma} u\right)_{Q_{r}\left(X_{0}\right)}\right| d x d t & \leq N k^{-1}\left(f_{Q_{k r}\left(X_{0}\right)}\left|D^{\gamma} u\right|^{2} d x d t\right)^{\frac{1}{2}} \\
& +N k^{m+\frac{d}{2}}\left(f_{Q_{k r}\left(X_{0}\right)}|f|^{2} d x d t\right)^{\frac{1}{2}}
\end{aligned}
$$

where $N=N(d, n, m, \delta)>0$.

REMARK 4.2. The system considered in [5] is formally different from our system. However, if we take into consideration the fact that every multi-index of length $2 m$ can be written as the sum of two multi-indices of length $m$, we obtain that all the hypotheses of Corollary 4.7 in [5] are verified. This last states that, for any $r \in(0, \infty), k \in[8, \infty), \lambda>0, X_{0}=\left(t_{0}, x_{0}\right) \in \mathbb{R}^{d+1}$ and $f \in L_{2, l o c}\left(\mathbb{R}^{d+1}\right)$, if $u \in W_{2, l o c}^{1,2 m}\left(\mathbb{R}^{d+1}\right)$ satisfies $u_{t}+(-1)^{m} L_{0} u+\lambda u=f$ in $Q_{k r}\left(X_{0}\right)$ then for any $\gamma$, $|\gamma|=2 m$ we have

$$
\begin{aligned}
& f_{Q_{r}\left(X_{0}\right)}\left|D^{\gamma} u-\left(D^{\gamma} u\right)_{Q_{r}\left(X_{0}\right)}\right| d x d t+\lambda \underset{Q_{r}\left(X_{0}\right)}{f}\left|u-(u)_{Q_{r}\left(X_{0}\right)}\right| d x d t \\
& \leq N k^{-1} \sum_{i=0}^{2 m} \lambda^{1-\frac{i}{2 m}}\left(\left|D^{i} u\right|^{2}\right)_{Q_{k r}\left(X_{0}\right)}^{\frac{1}{2}}+N k^{m+\frac{d}{2}}\left(|f|^{2}\right)_{Q_{k r}\left(X_{0}\right)}^{\frac{1}{2}}
\end{aligned}
$$

where $N=N(d, n, m, \delta)>0$. Then we can use it, after letting $\lambda \searrow 0$.

Improving the above theorem and following the hints to Exercises 4.3.5 and 4.3.6 in [12], we get the following two corollaries.

Corollary 4.3. Let $r \in(0, \infty), k \in[8, \infty), X_{0}=\left(t_{0}, x_{0}\right) \in \mathbb{R}^{d+1}$ and $f \in$ $L_{2, \text { loc }}\left(\mathbb{R}^{d+1}\right)$. Assume that $u \in W_{2, \text { loc }}^{1,2 m}\left(\mathbb{R}^{d+1}\right)$ satisfies (4.1). Then for any $\gamma,|\gamma|=2 m$, we have

$$
\begin{aligned}
f_{Q_{r}\left(X_{0}\right)}\left|D^{\gamma} u-\left(D^{\gamma} u\right)_{Q_{r}\left(X_{0}\right)}\right| d x d t & \leq N k^{-1}\left(f_{Q_{k r}\left(X_{0}\right)}\left|D^{\gamma} u\right|^{2} d x d t\right)^{\frac{1}{2}} \\
& +N k^{m+\frac{d}{2}}\left(f_{Q_{k r}\left(X_{0}\right)}\left|f-f_{B_{k r}\left(X_{0}\right)} f(t, y) d y\right|^{2} d x d t\right)^{\frac{1}{2}}
\end{aligned}
$$

where $N=N(d, n, m, \delta)>0$.

Proof. We consider a new function $v$ defined in the following way

$$
v(t, x)=u(t, x)-\int_{0}^{t} \underset{B_{k r}\left(X_{0}\right)}{f} f(s, x) d x d s .
$$


By the definition it follows that $v \in W_{2, l o c}^{1,2 m}\left(\mathbb{R}^{d+1}\right)$ and satisfies

$$
v_{t}+(-1)^{m} L_{0} v=f-f_{B_{k r}\left(X_{0}\right)} f(t, x) d x .
$$

Applying Theorem 4.1 to the function $v$, we obtain for any $\gamma,|\gamma|=2 m$,

$$
\begin{aligned}
\int_{Q_{r}\left(X_{0}\right)}\left|D^{\gamma} v-\left(D^{\gamma} v\right)_{Q_{r}\left(X_{0}\right)}\right| d x d t \leq N k^{-1}\left(f_{Q_{k r}\left(X_{0}\right)}\left|D^{\gamma} v\right|^{2} d x d t\right)^{\frac{1}{2}} \\
+N k^{m+\frac{d}{2}}\left(f_{Q_{k r\left(X_{0}\right)}}\left|f-\underset{B_{k r\left(X_{0}\right)}}{f} f(t, y) d y\right|^{2} d x d t\right)^{\frac{1}{2}}
\end{aligned}
$$

where $N=N(d, n, m, \delta)>0$. Again by the definition of $v$, it follows $D^{\gamma} v=D^{\gamma} u$ and so we, easily, have the result.

In the following corollary having the factor $k^{-1}$ in $(4.7)$, which can be made as small as we wish, will be crucial to obtain a Hölder space estimate.

Corollary 4.4. Let $r \in(0, \infty), k \in[8, \infty), X_{0}=\left(t_{0}, x_{0}\right) \in \mathbb{R}^{d+1}$, and $f \in$ $L_{2, \text { loc }}\left(\mathbb{R}^{d+1}\right)$. Assume that $u \in W_{2, \text { loc }}^{1,2 m}\left(\mathbb{R}^{d+1}\right)$ satisfies (4.1). Then for any $\gamma,|\gamma|=2 m$, we have

$$
\begin{aligned}
\int_{Q_{r}\left(X_{0}\right)}\left|D^{\gamma} u-\left(D^{\gamma} u\right)_{Q_{r}\left(X_{0}\right)}\right| d x d t & \\
& \leq N k^{-1}\left(f_{Q_{k r}\left(X_{0}\right)}\left|D^{\gamma} u-\left(D^{\gamma} u\right)_{Q_{k r}\left(X_{0}\right)}\right|^{2} d x d t\right)^{\frac{1}{2}} \\
& +N k^{m+\frac{d}{2}}\left(f_{Q_{k r}\left(X_{0}\right)}\left|f-f_{B_{k r}\left(X_{0}\right)} f(t, y) d y\right|^{2} d x d t\right)^{\frac{1}{2}}
\end{aligned}
$$

where $N=N(d, n, m, \delta)>0$.

Proof. We define a new function $v$ in the following way:

$$
v(t, x)=u(t, x)-\sum_{|\gamma|=2 m} B^{\gamma} x^{\gamma}
$$

where

$$
B^{\gamma}=\frac{1}{\gamma !} f_{Q_{k r}\left(X_{0}\right)} D^{\gamma} u(t, x) d t d x
$$

It is easy to see that $v \in W_{2, l o c}^{1,2 m}\left(\mathbb{R}^{d+1}\right)$ and $v$ satisfies

$$
v_{t}+(-1)^{m} L_{0} v=f-(-1)^{m} \sum_{|\gamma|=2 m} A^{\gamma} B^{\gamma} \gamma !
$$


Now apply Corollary 4.3 to the function $v$ and we get for any $\gamma,|\gamma|=2 m$,

$$
\begin{aligned}
f_{Q_{r}\left(X_{0}\right)} \mid D^{\gamma} v & -\left(D^{\gamma} v\right)_{Q_{r}\left(X_{0}\right)} \mid d x d t \leq N k^{-1}\left(f_{Q_{k r}\left(X_{0}\right)}\left|D^{\gamma} v\right|^{2} d x d t\right)^{\frac{1}{2}} \\
& +N k^{m+\frac{d}{2}}\left(f_{Q_{k r}\left(X_{0}\right)} \mid f-(-1)^{m} \sum_{|\gamma|=2 m} A^{\gamma} B^{\gamma} \gamma !\right. \\
& \left.-\left.\underset{B_{k r}\left(X_{0}\right)}{f}\left(f-(-1)^{m} \sum_{|\gamma|=2 m} A^{\gamma} B^{\gamma} \gamma !\right) d y\right|^{2} d x d t\right)^{\frac{1}{2}}
\end{aligned}
$$

where $N=N(d, n, m, \delta)>0$. By the definition of $v$ and taking into account that the average value of a constant on a set is the same constant, it follows

$$
\begin{aligned}
& D^{\gamma} v=D^{\gamma} u-B^{\gamma} \gamma !=D^{\gamma} u-\left(D^{\gamma} u\right)_{Q_{k r}\left(X_{0}\right)}, \\
& D^{\gamma} v-\left(D^{\gamma} v\right)_{Q_{r}\left(X_{0}\right)}=D^{\gamma} u-\left(D^{\gamma} u\right)_{Q_{r}\left(X_{0}\right)} .
\end{aligned}
$$

Combining the previous inequalities, we have the result.

5. A Schauder estimate for systems with coefficients independent of $x$. If $T=\infty$ the statement of the following lemma becomes a more general version of an exercise suggested by Krylov in [12] (see Chapter 10, Exercise 9).

Lemma 5.1. Assume that we have a function $u=u(t, x)$ of class $C^{\frac{\alpha}{2 m}, \alpha}\left(\mathbb{R}_{T}^{d+1}\right)$ such that for all $(t, x),(s, y) \in \mathbb{R}_{T}^{d+1}$ with finite $t, s$ and $\rho>0$, we have

$$
\begin{aligned}
& \int_{Q_{\rho}(t, x)} \int_{Q_{\rho}(s, y)} \mid u\left(r_{1}, p_{1}\right)-u\left(r_{2}, p_{2}\right) \mid d r_{1} d p_{1} d r_{2} d p_{2} \\
& \leq M\langle(t-s, x-y)\rangle^{\alpha+2 d+4 m}
\end{aligned}
$$

whenever

$$
4 \rho \leq\langle(t-s, x-y)\rangle, \quad Q_{\rho}(t, x), Q_{\rho}(s, y) \subset \mathbb{R}_{T}^{d+1},
$$

where $M$ is a constant. Then for all $(t, x),(s, y) \in \mathbb{R}_{T}^{d+1}$ it holds that

$$
|u(t, x)-u(s, y)| \leq N M\langle(t-s, x-y)\rangle^{\alpha},
$$

where $N=N(d, \alpha, n, m)$.

Proof. Define

$$
P=\sup _{\substack{(t, x),(s, y) \in \mathbb{R}_{T}^{d+1} \\(t, x) \neq(s, y)}} \frac{|u(t, x)-u(s, y)|}{\langle(t-s, x-y)\rangle^{\alpha}} .
$$

By the hypothesis about $u, P<\infty$. Take a $\rho>0$ and for $\left(r_{1}, p_{1}\right) \in Q_{\rho}(t, x)$, $\left(r_{2}, p_{2}\right) \in Q_{\rho}(s, y)$ write

$$
\begin{aligned}
& |u(t, x)-u(s, y)| \leq\left|u(t, x)-u\left(r_{1}, p_{1}\right)\right|+\left|u\left(r_{1}, p_{1}\right)-u\left(r_{2}, p_{2}\right)\right| \\
& +\left|u\left(r_{2}, p_{2}\right)-u(s, y)\right| \leq P 2^{\alpha+1} \rho^{\alpha}+\left|u\left(r_{1}, p_{1}\right)-u\left(r_{2}, p_{2}\right)\right| .
\end{aligned}
$$


Assuming that $\rho=\mu\langle(t-s, x-y)\rangle$ where the constant $\mu \in\left(0, \frac{1}{4}\right]$, we integrate through this estimate over $\left(r_{1}, p_{1}\right) \in Q_{\rho}(t, x)$ and $\left(r_{2}, p_{2}\right) \in Q_{\rho}(s, y)$ and due to (5.1) find

$$
\begin{gathered}
\rho^{4 m+2 d}|u(t, x)-u(s, y)| \leq P 2^{\alpha+1} \rho^{\alpha+2 d+4 m}+N M\langle(t-s, x-y)\rangle^{\alpha+2 d+4 m}, \\
|u(t, x)-u(s, y)| \leq\left(P 2^{\alpha+1} \mu^{\alpha}+M N \mu^{-2 d-4 m}\right)\langle(t-s, x-y)\rangle^{\alpha},
\end{gathered}
$$

where $N$ depends only on $d$, which by the definition of $P$ means that

$$
P \leq P 2^{\alpha+1} \mu^{\alpha}+M N \mu^{-2 d-4 m} .
$$

Finally, choose any $\mu$ so that not only $\mu \leq \frac{1}{4}$ but also $2^{\alpha+1} \mu^{\alpha} \leq \frac{1}{2}$ which is possible due to $\alpha>0$. So we obtain the result.

Proof of Theorem 2.1. Take a $\rho>0$ such that $4 \rho \leq\langle(t-s, x-y)\rangle$ and a multi-index $\gamma$ such that $|\gamma|=2 m$. For $(t, x),(s, y) \in \mathbb{R}_{T}^{d+1}$ write

$$
\begin{aligned}
& \int_{Q_{\rho}(t, x)} \int_{Q_{\rho}(s, y)}\left|D^{\gamma} u\left(r_{1}, p_{1}\right)-D^{\gamma} u\left(r_{2}, p_{2}\right)\right| d r_{1} d p_{1} d r_{2} d p_{2} \\
& \leq \int_{Q_{\rho}(t, x)} \int_{Q_{\rho}(s, y)}\left|D^{\gamma} u\left(r_{1}, p_{1}\right)-\left(D^{\gamma} u\right)_{Q_{r}(C)}\right| d r_{1} d p_{1} d r_{2} d p_{2} \\
& +\int_{Q_{\rho}(t, x)} \int_{Q_{\rho}(s, y)}\left|D^{\gamma} u\left(r_{2}, p_{2}\right)-\left(D^{\gamma} u\right)_{Q_{r}(C)}\right| d r_{1} d p_{1} d r_{2} d p_{2} \\
& \leq N r^{4 m+2 d} f_{Q_{r}(C)}\left|D^{\gamma} u\left(r_{3}, p_{3}\right)-\left(D^{\gamma} u\right)_{Q_{r}(C)}\right| d r_{3} d p_{3}
\end{aligned}
$$

where $N=N(d), Q_{r}(C)$ is a cylinder with radius $r=2\langle(t-s, x-y)\rangle$ and center $C=\left(s, \frac{x+y}{2}\right)$ if $s \geq t$ otherwise $C=\left(t, \frac{x+y}{2}\right)$. Using only the condition about $\rho$ and the definition of parabolic distance, one can easily check that $Q_{r}(C)$ is a subset of $\mathbb{R}_{T}^{d+1}$ which contains both $Q_{\rho}(t, x)$ and $Q_{\rho}(s, y)$.

Now, if we define $u(t, x):=u(T, x)$ for $t>T$, we can apply Corollary 4.4 to the extended function, which we still call $u$, to obtain

$$
\begin{aligned}
& \int_{Q_{r}(C)}\left|D^{\gamma} u\left(r_{3}, p_{3}\right)-\left(D^{\gamma} u\right)_{Q_{r}(C)}\right| d r_{3} d p_{3} \\
& \leq N_{1} k^{-1}\left(f_{Q_{k r(C)}}\left|D^{\gamma} u\left(r_{3}, p_{3}\right)-\left(D^{\gamma} u\right)_{Q_{k r(C)}}\right|^{2} d r_{3} d p_{3}\right)^{\frac{1}{2}} \\
& +N_{1} k^{m+\frac{d}{2}}\left(f_{Q_{k r(C)}}\left|f\left(r_{3}, p_{3}\right)-f_{B_{k r(C)}}\right|^{2} d r_{3} d p_{3}\right)^{\frac{1}{2}}
\end{aligned}
$$

where $N_{1}=N_{1}(d, n, m, \delta)$, and $k \in[8, \infty)$. Taking into consideration that the following estimates hold

$$
\left(\underset{Q_{k r(C)}}{f}\left|D^{\gamma} u\left(r_{3}, p_{3}\right)-\left(D^{\gamma} u\right)_{Q_{k r(C)}}\right|^{2} d r_{3} d p_{3}\right)^{\frac{1}{2}} \leq N_{2}(k r)^{\alpha}\left[D^{\gamma} u\right]_{\alpha, T}
$$




$$
\left(f_{Q_{k r(C)}}\left|f\left(r_{3}, p_{3}\right)-f_{B_{k r(C)}}\right|^{2} d r_{3} d p_{3}\right)^{\frac{1}{2}} \leq N_{3}(k r)^{\alpha} \sup _{r_{3} \leq T}[f]_{\alpha}\left(r_{3}\right),
$$

where $N_{2}$ and $N_{3}$ depend only on $\alpha$, by (5.6) and (5.7) we find that the function $D^{\gamma} u$ verifies (5.1) with constant

$$
M=N_{4} k^{\alpha-1}\left[D^{\gamma} u\right]_{\alpha, T}+N_{4} k^{m+\frac{d}{2}+\alpha} \sup _{r_{3} \leq T}[f]_{\alpha}\left(r_{3}\right),
$$

where $N_{4}=N_{4}(d, \alpha, n, m, \delta)$. Combining the hypothesis about the function $u$ with Corollary 3.3, we see that $D^{\gamma} u$ verifies the assumptions of Lemma 5.1. Then, applying it, we obtain

$$
\left[D^{\gamma} u\right]_{\alpha, T} \leq N_{5} k^{\alpha-1}\left[D^{\gamma} u\right]_{\alpha, T}+N_{5} k^{m+\frac{d}{2}+\alpha} \sup _{r_{3} \leq T}[f]_{\alpha}\left(r_{3}\right)
$$

where $N_{5}=N_{5}(d, \alpha, n, m, \delta)$. Finally, choose any $k$ so that not only $k \geq 8$ but also $N_{5} k^{\alpha-1}<\frac{1}{2}$, which is possible due to $0<\alpha<1$, we have the result.

6. Schauder estimates in the general case. From now on, we assume that all coefficients depend on $t$ and $x$. We recall the following well-known lemma that is proved for scalar-valued functions in [11] (see Section 4.1).

Lemma 6.1. Fix a cut-off function $\zeta \in C_{0}^{\infty}\left(\mathbb{R}^{d}\right)$ such that $\zeta(x)=1$ for $|x| \leq 1$ and $\zeta(x)=0$ for $|x| \geq 2$ and $0 \leq \zeta \leq 1$, and fix an integer $r$. Then there is a constant $N=N(d, r, n, \theta)$ such that for any $\theta \in[0,1], R \geq 1$, finite $t \leq T$ and $u(t, \cdot) \in C^{r+\theta}\left(\mathbb{R}^{d}\right)$ we have

$$
|u|_{r+\theta}(t) \leq N \sup _{y \in \mathbb{R}^{d}}\left(\left[u \zeta_{R}^{y}\right]_{r+\theta}(t)+\left|u \zeta_{R}^{y}\right|_{0}(t)\right),
$$

where $\zeta_{R}^{y}(x)=\zeta\left(R^{-1}(x-y)\right)$.

Before proceeding, we recall an inequality which will be used in the proof of Theorem 2.4. If $u(t, \cdot), v(t, \cdot) \in C^{\alpha}\left(\mathbb{R}^{d}\right)$ for any $t \leq T$ then

$$
[u v]_{\alpha}(t) \leq|u|_{0}(t)[v]_{\alpha}(t)+|v|_{0}(t)[u]_{\alpha}(t) .
$$

Proof of Theorem 2.4. Take a constant $R \geq 1$, the function $\zeta$ from Lemma 6.1, take a point $y \in \mathbb{R}^{d}$ and finite $s \leq T$. We use the idea of freezing the coefficients to obtain, by Theorem 2.1,

$$
\left[u \zeta_{R}^{y}\right]_{2 m+\alpha}(s) \leq N \sup _{t \leq T}\left(\left[\left(u \zeta_{R}^{y}\right)_{t}+(-1)^{m} L_{0}(t, y)\left(u \zeta_{R}^{y}\right)\right]_{\alpha}(t)\right),
$$

where $N=N(d, \alpha, n, m, \delta)$. After evaluating $L_{0}(t, y)\left(u(t, x) \zeta_{R}^{y}(x)\right)$ and applying inequality (6.2), we obtain

$$
\begin{aligned}
& {\left[\left(u \zeta_{R}^{y}\right)_{t}+(-1)^{m} L_{0}(t, y)\left(u \zeta_{R}^{y}\right)\right]_{\alpha}(t) \leq\left[\zeta_{R}^{y}\left(u_{t}+(-1)^{m} L_{0}(t, y) u\right)\right]_{\alpha}(t)} \\
& +N R^{-1}|u|_{2 m-1+\alpha}(t) \leq\left[\zeta_{R}^{y} f\right]_{\alpha}(t)+\left[\zeta_{R}^{y}\left\{L_{0}(t, y)-L_{0}\right\} u\right]_{\alpha}(t) \\
& +N R^{-1}|u|_{2 m+\alpha}(t)
\end{aligned}
$$


Moreover,

$$
\begin{gathered}
{\left[\zeta_{R}^{y} f\right]_{\alpha}(t) \leq[f]_{\alpha}(t)+N R^{-\alpha}|f|_{0}(t)} \\
{\left[\zeta_{R}^{y}\left\{L_{0}(t, y)-L_{0}\right\} u\right]_{\alpha}(t) \leq N \max _{|\gamma|=2 m}\left|\zeta_{R}^{y}\left\{A^{\gamma}(t, y)-A^{\gamma}\right\}\right|_{0}(t) \cdot|u|_{2 m+\alpha}(t)} \\
+N[u]_{2 m}(t)\left\{\left[\zeta_{R}^{y}\right]_{\alpha}(t)+\max _{|\gamma|=2 m}\left[A^{\gamma}\right]_{\alpha}(t)\right\} \leq N R^{\alpha}|u|_{2 m+\alpha}(t) \\
+N\left(R^{-\alpha}+K\right)[u]_{2 m}(t) .
\end{gathered}
$$

By (6.6), applying an interpolation inequality (see Theorem 3.2.1 in [11]), we get

$$
\begin{aligned}
{\left[\zeta_{R}^{y}\left\{L_{0}(t, y)-L_{0}\right\} u\right]_{\alpha}(t) } & \leq N\left(R^{\alpha}+R^{-\alpha} \varepsilon^{\alpha}+\varepsilon^{\alpha}\right)|u|_{2 m+\alpha}(t) \\
& +N\left(R^{-\alpha} \varepsilon^{-2 m}+\varepsilon^{-2 m}\right)|u|_{0}(t)
\end{aligned}
$$

for all $\varepsilon>0$.

Coming back to $(6.3)$ we get

$$
\begin{aligned}
{\left[u \zeta_{R}^{y}\right]_{2 m+\alpha}(s) } & \leq N \sup _{t \leq T}\left(N R^{-\alpha}|f|_{0}(t)+[f]_{\alpha}(t)+c_{1}(R, \varepsilon)|u|_{2 m+\alpha}(t)\right. \\
& \left.+c_{2}(R, \varepsilon)|u|_{0}(t)\right)
\end{aligned}
$$

which by Lemma 6.1 implies that

$$
\begin{aligned}
|u|_{2 m+\alpha}(s) & \leq N \sup _{t \leq T}\left([f]_{\alpha}(t)+N R^{-\alpha}|f|_{0}(t)+c_{1}(R, \varepsilon)|u|_{2 m+\alpha}(t)\right. \\
& \left.+c_{2}(R, \varepsilon)|u|_{0}(t)\right) .
\end{aligned}
$$

One can choose first $R$ and then $\varepsilon$ such that

$$
N \cdot c_{1}(R, \varepsilon) \leq \frac{1}{2}
$$

Using (6.10), from (6.9), it follows

$$
\sup _{s \leq T}|u|_{2 m+\alpha}(s) \leq N \sup _{t \leq T}\left([f]_{\alpha}(t)+N|f|_{0}(t)+N|u|_{0}(t)\right) \cdot \square
$$

As consequence of the previous theorem, we obtain a Hölder estimate for the solutions of system with non-zero lower-order terms.

COROLlary 6.2. Let $u \in \mathcal{C}^{2 m+\alpha}(T)$ satisfy (1.1). Then there exists a constant $N=N(K, d, \alpha, n, m, \delta)$ such that

$$
\sup _{s \leq T}|u|_{2 m+\alpha}(s) \leq N \sup _{t \leq T}\left([f]_{\alpha}(t)+|f|_{0}(t)+|u|_{0}(t)\right) .
$$

Proof. Considering the fact that we can write our system in the form

$$
u_{t}+(-1)^{m} L_{0} u=f+(-1)^{m} \sum_{|\gamma|<2 m} A^{\gamma} D^{\gamma} u
$$


and then using Theorem 2.4, we get

$$
\begin{aligned}
& \sup _{s \leq T}|u|_{2 m+\alpha}(s) \\
& \leq \sup _{t \leq T}\left([f]_{\alpha}(t)+\sum_{|\gamma|<2 m}\left(\left[A^{\gamma} D^{\gamma} u\right]_{\alpha}(t)+\left[A^{\gamma} D^{\gamma} u\right]_{0}(t)\right)+|f|_{0}(t)+|u|_{0}(t)\right),
\end{aligned}
$$

where $N=N(K, d, \alpha, n, m, \delta)$. As always, using (6.2) and some interpolation inequalities, the additional terms on the right can be estimated through $\varepsilon|u|_{2 m+\alpha}(t)$ plus $N(\varepsilon)|u|_{0}(t)$; and then by choosing small $\varepsilon$, collecting like terms we arrive at (6.12). The corollary is proved.

7. An existence result. Let $T \in(0, \infty)$ and $f$ be in the space $\mathscr{C}^{\alpha}(0, T)$ of all measurable functions defined on $[0, T] \times \mathbb{R}^{d}$ such that

$$
\|f\|_{\mathscr{C}^{\alpha}(0, T)}:=\sup _{0 \leq t \leq T}|f|_{\alpha}(t)<\infty .
$$

The aim of this section is to prove the unique solvability in a suitable space of the problem

$$
\begin{cases}u_{t}+(-1)^{m} L u=f & \text { in }(0, T) \times \mathbb{R}^{d} \\ u(0, x)=0 & \text { in } \mathbb{R}^{d} .\end{cases}
$$

In order to do this, we start to solve the same system in a smaller domain. To be more precise, let $\varepsilon$ be a small positive number. We consider the following problem:

$$
u_{t}+(-1)^{m} L u=f \quad \text { in }(0, \varepsilon) \times \mathbb{R}^{d} .
$$

Introduce the space $\mathscr{C}^{2 m+\alpha}(0, \varepsilon)$ as a subspace of $\mathcal{C}^{2 m+\alpha}(0, \varepsilon)$ such that $u(0, x)=$ 0 for any $x \in \mathbb{R}^{d}$ and

$$
\|u\|_{\mathscr{C}^{2 m+\alpha}(0, \varepsilon)}:=\sup _{0 \leq t \leq \varepsilon}\left|u_{t}\right|_{\alpha}(t)+\sup _{0 \leq t \leq \varepsilon}|u|_{2 m+\alpha}(t)<\infty
$$

One can easily check that $\mathscr{C}^{\alpha}(0, T)$ and $\mathscr{C}^{2 m+\alpha}(0, \varepsilon)$ are Banach spaces.

THEOREM 7.1. There exists $\varepsilon>0$ such that, for any $f \in \mathscr{C}^{\alpha}(0, \varepsilon)$, system (7.3) admits a unique solution $u \in \mathscr{C}^{2 m+\alpha}(0, \varepsilon)$.

Proof. Let $\varepsilon$ be a positive number lesser than $T$ which we will specify later. At first suppose that such solution $u \in \mathscr{C}^{2 m+\alpha}(0, \varepsilon)$ of $(7.3)$ exists. We define $u(t, x)$ for negative $t$ as zero, $f(t, x)$ for $t<0$ and $t>\varepsilon$ as zero and we still call $u$ and $f$ the extended functions. By the definition, the function $u$ belongs to $\mathcal{C}^{2 m+\alpha}(\varepsilon)$ and satisfies

$$
u_{t}+(-1)^{m} L u=f \quad \text { in } \mathbb{R}_{\varepsilon}^{d+1} .
$$

By Corollary 6.2 we get the a priori estimate

$$
\sup _{0 \leq t \leq \varepsilon}|u|_{2 m+\alpha}(t) \leq N \sup _{0 \leq t \leq \varepsilon}\left([f]_{\alpha}(t)+|f|_{0}(t)+|u|_{0}(t)\right)
$$

where $N=N(K, d, \alpha, n, m, \delta)$. 
Now, Newton-Leibnitz formula allows us to estimate the last term in the right hand side of (7.6) in the following way

$$
\sup _{0 \leq t \leq \varepsilon}|u|_{0}(t) \leq \varepsilon \sup _{0 \leq t \leq \varepsilon}\left|u_{t}\right|_{0}(t) .
$$

From system (7.3) we get an estimate of $\left|u_{t}\right|_{\alpha}(t)$ in terms of $[f]_{\alpha}(t)$ and $|f|_{0}(t)$. In fact

$$
\sup _{0 \leq t \leq \varepsilon}\left|u_{t}\right|_{0}(t) \leq \sup _{0 \leq t \leq \varepsilon}\left(|f|_{0}(t)+N|u|_{2 m}(t)\right)
$$

and

$$
\sup _{0 \leq t \leq \varepsilon}\left[u_{t}\right]_{\alpha}(t) \leq \sup _{0 \leq t \leq \varepsilon}\left([f]_{\alpha}(t)+N|u|_{2 m+\alpha}(t)\right) .
$$

Then, using again Corollary 6.2,

$$
\sup _{0 \leq t \leq \varepsilon}\left|u_{t}\right|_{\alpha}(t) \leq N \sup _{0 \leq t \leq \varepsilon}\left([f]_{\alpha}(t)+|f|_{0}(t)+|u|_{0}(t)\right) .
$$

Moreover, from (7.7), using (7.8), we have

$$
\sup _{0 \leq t \leq \varepsilon}|u|_{0}(t) \leq \sup _{0 \leq t \leq \varepsilon}\left(\varepsilon|f|_{0}(t)+N \varepsilon|u|_{2 m+\alpha}(t)\right)
$$

Combining such estimate with (7.6) and (7.10), we get

$$
\sup _{0 \leq t \leq \varepsilon}\left|u_{t}\right|_{\alpha}(t)+\sup _{0 \leq t \leq \varepsilon}|u|_{2 m+\alpha}(t) \leq N \sup _{0 \leq t \leq \varepsilon}\left(c(\varepsilon)|f|_{\alpha}(t)+\varepsilon|u|_{2 m+\alpha}(t)\right) .
$$

Choosing $\varepsilon$ such that $\varepsilon<\min \left\{\frac{1}{2 N}, T\right\}$ we deduce

$$
\|u\|_{\mathscr{C}^{2 m+\alpha}(0, \varepsilon)} \leq N\|f\|_{\mathscr{C}^{\alpha}(0, \varepsilon)}
$$

where $N=N(K, d, \alpha, n, m, \delta)$.

Now we are interested in the proof of the existence of a unique solution $u$ which solves (7.3). Uniqueness obviously follows from this last estimate. To prove existence, we use the method of continuity. For $\tau \in[0,1]$ define

$$
L^{\tau}=\tau L+(1-\tau) \Delta^{2 m}
$$

where $\Delta^{2 m}$ denotes the operator with coefficients $A^{\gamma}=\delta_{\gamma} I_{n \times n}$, and let $Z$ be the set of all points $\tau \in[0,1]$ for which the statement of the theorem is true (with $L^{\tau}$ in place of $L$ ).

First we want to prove that $Z$ is not empty. Our aim is to find a unique function $u$ in $\mathscr{C}^{2 m+\alpha}(0, \varepsilon)$ which solves the system

$$
u_{t}+(-1)^{m} \Delta^{2 m} u=f \quad \text { in }(0, \varepsilon) \times \mathbb{R}^{d} .
$$

Observe that if $f$ is Hölder continuous also in time, the proof of this fact becomes well known.

In our case, let $\Phi(t)$ be a smooth compactly supported function in $\mathbb{R}$, with $\int_{\mathbb{R}} \Phi(t) d t=1$. For any $\nu>0$ and $(t, x) \in \mathbb{R}^{d+1}$ we introduce the function

$$
f^{(\nu)}(t, x)=\left(f(\cdot, x) * \Phi_{\nu}\right)(t)
$$


where

$$
\Phi_{\nu}(t)=\frac{1}{\nu^{2 m}} \Phi\left(\frac{t}{\nu^{2 m}}\right) .
$$

For any $\nu$, the functions $f^{(\nu)}$ are Hölder continuous in both variables. Then, by a classical result (see Theorem $4^{\prime} .3$ of [6]), we get that there exists a unique function $u^{(\nu)}$, with all derivatives of order less or equal than $2 m$ Hölder continuous in $t$ and in $x$, solution of the system

$$
\left(u^{(\nu)}\right)_{t}+(-1)^{m} \Delta^{2 m} u^{(\nu)}=f^{(\nu)} \quad \text { in }(0, \varepsilon) \times \mathbb{R}^{d},
$$

and that satisfies the initial zero condition. Obviously, for such function, the a priori estimate

$$
\left\|u^{(\nu)}\right\|_{\mathscr{C}^{2 m+\alpha}(0, \varepsilon)} \leq N|| f^{(\nu)} \|_{\mathscr{C}^{\alpha}(0, \varepsilon)}
$$

holds with $N$ independent of $f$ and $\varepsilon$. This estimate shows that the family $u^{(1 / j)}$, $D^{1} u^{(1 / j)}, D^{2} u^{(1 / j)}, \ldots, D^{2 m} u^{(1 / j)}$ is uniformly bounded and equicontinuous on any bounded subset of $[0, \varepsilon] \times \mathbb{R}^{d}$. Then, by Ascoli-Arzelà theorem and Theorem 7.17 in [18], there are a continuous function $u$ on $[0, \varepsilon] \times \mathbb{R}^{d}$ having bounded and continuous derivatives with respect to $x$ up to the order $2 m$ and a sequence $j_{n} \rightarrow \infty$ such that

$$
\left(u^{\left(1 / j_{n}\right)}, D^{1} u^{\left(1 / j_{n}\right)}, D^{2} u^{\left(1 / j_{n}\right)}, \ldots, D^{2 m} u^{\left(1 / j_{n}\right)}\right) \longrightarrow\left(u, D^{1} u, D^{2} u, \ldots, D^{2 m} u\right)
$$

as $j \rightarrow \infty$, uniformly on bounded subsets of $[0, \varepsilon] \times \mathbb{R}^{d}$. Passing to the limit in equation (7.18) (of course, in the integral form, see (2.6)), corresponding to $u^{\left(1 / j_{n}\right)}$ we obtain that $u$ satisfies (7.15). The fact that $u \in \mathscr{C}^{2 m+\alpha}(0, \varepsilon)$ follows by estimate (7.19) which holds with the same $N$ with $u$ in place of $u^{(1 / j)}$. So we obtain the desidered result.

Obviously estimate (7.13) holds with the same constant $N$ for all solutions of the equation $u_{t}+(-1)^{m} L^{\tau} u=f$. This implies that $Z$ is closed. Therefore, to finish the proof it remains only to prove that $Z$ is open in the topology of $[0,1]$. Take any point $\tau_{0}$ in $Z \cap[0,1]$ and define the linear operator

$$
R: \mathscr{C}^{\alpha}(0, \varepsilon) \longrightarrow \mathscr{C}^{2 m+\alpha}(0, \varepsilon)
$$

such that it takes any $f \in \mathscr{C}^{\alpha}(0, \varepsilon)$ into the set of functions $\mathscr{C}^{2 m+\alpha}(0, \varepsilon)$ which solve $u_{t}+(-1)^{m} L^{\tau_{0}} u=f$. By the assumption $R$ is well defined and by (7.13) is bounded.

Now in order to show that for $\tau \in[0,1]$ close to $\tau_{0}$ the system $u_{t}+(-1)^{m} L^{\tau} u=f$ is solvable, we write this system as

$$
u_{t}+(-1)^{m} L^{\tau_{0}} u=f+(-1)^{m}\left(L^{\tau_{0}}-L^{\tau}\right) u
$$

or, equivalently,

$$
u=R f+R\left[(-1)^{m}\left(L^{\tau_{0}}-L^{\tau}\right)\right] u,
$$

and we show that the operator $R\left[(-1)^{m}\left(L^{\tau_{0}}-L^{\tau}\right)\right]$ is a contraction in $\mathscr{C}^{\alpha}(0, \varepsilon)$ for all $\tau$ close to $\tau_{0}$.

By the above for certain constants $N$ independent of $\tau, u$

$$
\begin{aligned}
& \left\|R\left[(-1)^{m}\left(L^{\tau_{0}}-L^{\tau}\right)\right] u\right\|_{\mathscr{C}^{2 m+\alpha}(0, \varepsilon)} \leq N||\left(L^{\tau_{0}}-L^{\tau}\right) \|_{\mathscr{C}^{\alpha}(0, \varepsilon)} \\
& =N\left|\tau_{0}-\tau\right|\left|\left(L-\Delta^{2 m}\right)\right|_{\mathscr{C}^{\alpha}(0, \varepsilon)} \leq N_{1}\left|\tau_{0}-\tau\right|\|u\|_{\mathscr{C}^{2 m+\alpha}(0, \varepsilon)} .
\end{aligned}
$$


For $\tau$ such that $N_{1}\left|\tau_{0}-\tau\right| \leq \frac{1}{2}$, the operator $R\left(L^{\tau_{0}}-L^{\tau}\right)$ is indeed a contraction and the theorem is proved.

Our next result concerns the Cauchy problem with non-zero initial condition.

Corollary 7.2. Assume that we are given a function $\psi \in C^{2 m+\alpha}\left(\mathbb{R}^{d}\right)$. Let $\varepsilon$ the positive number which comes out from Theorem 7.1. Then for any $f \in \mathscr{C}^{\alpha}(0, \varepsilon)$ there exists a unique $u \in \mathcal{C}^{2 m+\alpha}(0, \varepsilon)$ satisfying (7.3) and such that $u(0, x)=\psi(x)$ for any $x \in \mathbb{R}^{d}$.

Proof. From Theorem 7.1 we know that there exists a unique solution $v \in$ $\mathscr{C}^{2 m+\alpha}(0, \varepsilon)$ of the problem

$$
v_{t}+(-1)^{m} L v=\tilde{f}
$$

with $\tilde{f}=f-(-1)^{m} L \psi$ in $\mathscr{C}^{\alpha}(0, \varepsilon)$.

Now, if we consider the function $u(t, x)=v(t, x)+\psi(x)$, we easily see that $u \in \mathcal{C}^{2 m+\alpha}(0, \varepsilon)$ is a solution of $(7.3)$ and satisfies $u(0, x)=\psi(x)$ for any $x \in \mathbb{R}^{d}$.

The uniqueness of such solution follows by the uniqueness of (7.24). (7.2).

Finally we conclude explaining as the above results can be used to solve problem

Let $f \in \mathscr{C}^{\alpha}(0, T), \varepsilon$ be the positive number which comes out from Theorem 7.1, $f_{\mid[0, \varepsilon]}$ be the right-hand side of equation $(7.3)$ and $u$ be in $\mathscr{C}^{2 m+\alpha}(0, \varepsilon)$ the corresponding solution of such equation. Consider the following problem:

$$
\left\{\begin{array}{l}
v_{t}+(-1)^{m} L v=f_{\mid[\varepsilon, 2 \varepsilon] \quad \text { in }(\varepsilon, 2 \varepsilon) \times \mathbb{R}^{d},}, \\
v(\varepsilon, x)=u(\varepsilon, x) \quad \text { in } \mathbb{R}^{d} .
\end{array}\right.
$$

Repeating the argument of Corollary 7.2, replacing, in the definitions, $(0, \varepsilon)$ with $(\varepsilon, 2 \varepsilon)$, we obtain the existence of a unique function $v$ in $\mathcal{C}^{2 m+\alpha}(\varepsilon, 2 \varepsilon)$ solution of $(7.25)$.

Thus, after a finite number of steps, we obtain a unique solution $u$ in $\mathscr{C}^{2 m+\alpha}(0, T)$ which solves problem (7.2). Such solution is built as combination, through characteristics functions, of the solutions in the smaller domains. The fact that this solution is just in the space $\mathscr{C}^{2 m+\alpha}(0, T)$ is guaranteed by the required initial conditions and by the integral form (2.6) of the systems considered in the single Cauchy problems. The uniqueness follows by the uniqueness of the single solutions.

So we have proved the following.

TheOREM 7.3. For any $f$ in $\mathscr{C}^{\alpha}(0, T)$, there exists a unique $u$ in $\mathscr{C}^{2 m+\alpha}(0, T)$, solution of (7.2).

REMARK 7.4. Taking into account Corollary 3.3, we can also state that the derivatives $D^{2 m} u$ of the solution $u$ that comes out from the last theorem are Hölder continuous in $t$ with exponent $\frac{\alpha}{2 m}$. Owing to the lack of regularity of the coefficients with respect to the variable $t$, we do not expect the solution to problem (7.2) to be continuously $t$-differentiable in the whole of $[0, T] \times \mathbb{R}^{d}$ even if the coefficients are smooth with respect to $x$. Nevertheless, the discontinuity of the coefficients does not influence the regularity of the solution with respect to the space variables. 


\section{REFERENCES}

[1] R. B. BARrar, Some estimates for solution of parabolic equations, J. Math. Anal. Appl., 3 (1961), pp. 373-397.

[2] A. BRANDT, Interior Schauder estimates for parabolic differential- (or difference-) equations via the maximum principle, Israel J. Math., 7 (1969), pp. 254-262.

[3] S. Byun, Hessian estimates in Orlicz spaces for fourth-order parabolic systems in non-smooth domains, J. Differential Equations, 246 (2009), pp. 3518-3534.

[4] S. Campanato, Equazioni paraboliche del secondo ordine e spazi $\mathcal{L}^{2, \theta}(\Omega, \delta)$, Ann. Mat. Pura Appl., 73 (1966), pp. 55-102.

[5] H. Dong AND D. KIM, On the $L^{p}$-solvability of higher order parabolic and elliptic systems with BMO coefficients, Arch. Rational Mech. Anal., 199 (2011), pp. 889-941.

[6] S. D. Ejdel'man, Parabolic Systems, North-Holland, Amsterdam, London, 1969.

[7] A. FRIEDman, Boundary estimates for second order parabolic equations and their applications, J. Math. Mech., 7 (1958), pp. 771-792.

[8] A. Friedman, Partial differential equations of parabolic type, Prentice-Hall, Englewood Cliffs, N.J., 1964.

[9] M. Giaquinta, Introduction to regularity theory for nonlinear elliptic systems, Lectures in Mathematics ETH Zürich. Birkhäuser Verlag, Basel, 1993.

[10] B. KNERR, Parabolic interior Schauder estimates by the maximum principle, Arch. Rational Math. Anal., 75 (1980), pp. 51-58.

[11] N. V. KRYLOv, Lectures on elliptic and parabolic equations in Hölder spaces, American Mathematical Society, Providence, RI, 1996.

[12] N. V. KRYLOV, Lectures on elliptic and parabolic equations in Sobolev spaces, American Mathematical Society, Providence, RI, 2008.

[13] N. V. KRylov And E. PRIOLA, Elliptic and parabolic second-order PDEs with growing coefficients, Comm. Partial Differential Equations, 35 (2010), pp. 1-22.

[14] N. V. KRYlov And M. V. SAFOnov, Certain properties of solutions of parabolic equations with measurable coefficients, Izv. Akad. Nauk, 44 (1980), pp. 161-175 (Russian); English transl. in Math. USSR-Izv., 16 (1981), pp. 155-164.

[15] S. LeOnARdi, J. Kottas, And J. Stara, Hölder regularity of the solutions of some classes of elliptic systems in convex nonsmooth domains, Nonlinear Anal., 60 (2005), pp. 925-944.

[16] G. M. LIEBERMAN, Intermediate Schauder theory for second order parabolic equations IV. Time irregularity and regularity, Differential Integral Equations, 5 (1992), pp. 1219-1236.

[17] D. Palagachev And L. Softova, A priori estimates and precise regularity for parabolic systems with discontinuous data, Discrete Contin. Dyn. Syst., 13 (2005), pp. 721-742.

[18] W. Rudin, Principles of mathematical analysis, McGraw-Hill Book Company, Inc., New YorkToronto-London, 1953.

[19] M. V. SAfonov, On the classical solvability of Bellman's elliptic equations, Dokl. Akad. Nauk SSSR, 278 (1984), pp. 810-813 (Russian); English transl. in Soviet. Math. Dokl., 30 (1984), pp. $482-485$.

[20] W. Schlag, Schauder and $L^{p}$ estimates for parabolic systems via Campanato spaces, Comm. Partial Differential Equations, 21 (1996), pp. 1141-1175.

[21] V. A. Solonnikov, On boundary value problems for linear parabolic systems of differential equations of general form, (Russian), Trudy Mat. Inst. Steklov., 83 (1965), pp. 3-163; English translation: Proceedings of the Steklov Institute of Mathematics. No 83 (1965): Boundary value problems of mathematical physics. III. Edited by O. A. Ladyženskaja. Translated from the Russian by A. Jablonskii, American Mathematical Society, Providence, R.I. 1967 iv +184 pp. 
S. BOCCIA 